\begin{tabular}{|l|l|}
\hline Postprint Version & 1.0 \\
\hline Journal website & http://archinte.ama-assn.org/cgi/reprint/171/2/181 \\
\hline Pubmed link & $\underline{\text { http://www.ncbi.nlm.nih.gov/pubmed?term=21263112 }}$ \\
\hline DOI & $10.1001 /$ archinternmed.2010.505 \\
\hline
\end{tabular}

This is a NIVEL certified Post Print, more info at http://www.nivel.eu

\title{
Where Do Diagnostic Adverse Events Come From?
}

\author{
ELLIS LADER, MD
}

Author Affiliations: Mid-Valley Cardiology, Kingston, New York, and Division of Cardiology, Department of Medicine, New York University School of Medicine, New York.

Correspondence: Dr Lader, Mid-Valley Cardiology, 111 Mary's Ave, Ste 3, Kingston, NY 12401 (ellis.lader @primecarepc.com).

I read with interest the article by Zwaan et $\mathrm{al}^{1}$ analyzing the causes of diagnostic adverse events (DAEs). They conclude that "knowledge-based mistakes" were the most common, but I believe that the real issues lie hidden behind the otherwise compelling statistics developed in the article.

We live in a setting where we increasingly see medicine practiced by protocol. That is not to say that compiling best practice guidelines or evidence-based algorithms are bad, but what it encourages is a lack of "creative thinking" when seeing patients and more of a "cookbookapproach." Couple this with the increasing pressures to see more patients in a shorter period, to get patients out of the hospital faster, and to enforce a conformity of therapy in the name of "comparative effectiveness," andwesee DAEs because practitioners just are not encouraged to think creatively.

Many mid-level health care providers are trained to deal with problems by rote and not by constructing differential diagnoses and may also be an increasing source of DAEs.

Multiple "handoffs" of care, most dramatically between primary care practitioners and hospitalists, allow key pieces of baseline clinical history to be omitted, setting the stage for another (and not negligible) form of DAE. And finally, we need to accept that all physicians are not equally smart.

When faced with palpitations and flushing, one physician may order a 24-hour urine screen for metanephrines and diagnose a pheochromocytoma, while another may pass off the symptoms as anxiety.

The solutions here are not simple. They start in the medical schools, ensuring that qualified applicants are accepted and ensuring careful training in critical and analytical thinking. Practitioners need time and complete and valid data to practice properly. They need to know the best and most current evidence in their fields. Maybe they need more protocols, but they need to know when not to use them.

1. Zwaan L, de Bruijne M, Wagner C, et al. Patient record review of the incidence, consequences, and causes of diagnostic adverse events. Arch Intern Med. 2010;170(12):1015-1021.

\section{IN REPLY}

Laura ZWaAn, MSc Martine de Bruijne, MD, PhD Cordula Wagner, PhD Abel ThiJs, MD, PhD MARleEn SMits, PhD Gerrit VAN DER WAL, MD, PhD DANiËLle R. M. TimMERMANS, PhD

Author Affiliations: Department of Public and Occupational Health, EMGO Institute for Health and Care research (Ms Zwaan and Drs de Bruijne, Wagner, van der Wal, and Timmermans), and Department of Internal Medicine (Dr Thijs),VU University Medical Center, Amsterdam, the Netherlands; and NIVEL, Netherlands Institute for Health Services Research, Utrecht (Drs Wagner and Smits).

Correspondence: Ms Zwaan, Emgo Institute for Health and Care Research, Public \& Occupational Health, Van der Boechorststraat 7, Amsterdam 1081BT, the Netherlands (laura.zwaan@vumc.nl). 
We thank Dr Lader for his interest in our article and his valuable insights. We agree that there is more to it than just knowledge-based mistakes underlying the occurrence of DAEs. The causes described by Dr Lader constitute plausible reasons that could contribute to the occurrence of DAEs.

We agree that evidence-based algorithms should not be followed blindly. Evidence-based algorithms represent the latest scientific findings and are therefore a valuable tool for diagnostic reasoning. However, as they are based on data from a large number of patients, these algorithms are not applicable to every patient and in every situation. It is thus particularly important for physicians to recognize the situations in which algorithms are not applicable. This implies that physicians, while diagnosing a patient, need to be aware of the whole context and should reflect on their thinking at the same time. ${ }^{1}$ For example, physicians are inclined to only look for data confirming their initial diagnosis. While this is often a good strategy, since it allows focusing on the most important examinations and tests, it sometimes leads to a diagnostic error, in particular when a patient reports symptoms not typical for the underlying disease. While making a diagnosis, physicians are not only bounded by limited cognitive capabilities, typical for the human being, but also by time and other constraints. This implies, and we believe this is in line with Dr Lader's remarks, that it is particularly important to study diagnostic reasoning in diverse situations with time and other constraints.

Although the study that we conducted provided interesting insights in the causes of DAEs, record reviews are limited in revealing more specific causal factors. Our study revealed that coordination between health care professionals and transfer of knowledge played a role in the occurrence of DAEs, but it was not possible to examine those causes in more detail. Further research is needed that focuses on the underlying reasons and mechanisms of why DAEs occur, taking into account the circumstances under which these diagnoses are made, eg, a study proposed by our research group. ${ }^{2}$ At the same time, we believe that critical thinking and other metacognitive skills ${ }^{1}$ may be incorporated in the medical curriculum. These techniques are used for training in the US army, another field where missing a relevant cue might be fatal.

1. Cohen MS, Freeman JT, Thompson BT. Training the naturalistic decision maker.

In: Zsambok CE, Klein G, eds. Naturalistic Decision Making. Mahwah, NJ: Erlbaum; 1997:257-268.

2. Zwaan L, Thijs A, Wagner C, van der Wal G, Timmermans DRM. Design of a study on suboptimal cognitive acts in the diagnostic process, the effect on patient outcomes and the influence of workload, fatigue and experience of physician. BMC Health Serv Res. 2009;9:65. 